POS PROCEEDINGS

\title{
Development of Radiation Damage Models for Irradiated Silicon Sensors Using TCAD Tools
}

\author{
Ranjeet Dalal ${ }^{1}$ \\ ${ }^{1}$ University of Delhi \\ Department of Physics, University of Delhi, India \\ E-mail: rdalalecern.ch
}

\begin{abstract}
A. Bhardwaj ${ }^{1}$, R. Eber ${ }^{2}$, T. Eichhorn ${ }^{3}$, K. Lalwani ${ }^{1}$, A. Messineo ${ }^{4}$, T. Peltola ${ }^{5}$, M. $^{2}$ Printz $^{2}$, K. Ranjan ${ }^{1}$

${ }^{I}$ University of Delhi, India ${ }^{2}$ Karlsruhe Institute of Technology, Germany ${ }^{3}$ Deutsches ElektronenSynchrotron, Germany ${ }^{4}$ Università di Pisa \& INFN sez. di Pisa, Italy ${ }^{5}$ Helsinki Institute of Physics, Finland
\end{abstract}

\begin{abstract}
During the high luminosity upgrade of the LHC (HL-LHC) the CMS tracking system will face a more intense radiation environment than the present system was designed for. In order to design radiation tolerant silicon sensors for the future CMS tracker upgrade it is fundamental to complement the measurement with device simulation. This will help in both the understanding of the device performance and in the optimization of the design parameters. One of the important ingredients of the device simulation is to develop a radiation damage model incorporating both bulk and surface damage. In this paper we will discuss the development of a radiation damage model by using commercial TCAD packages (Silvaco and Synopsys), which successfully reproduce the recent measurements like leakage current, depletion voltage, interstrip capacitance and interstrip resistance, and provides an insight into the performance of irradiated silicon strip sensors.
\end{abstract}

Technology and Instrumentation in Particle Physics 2014

2-6 June, 2014

Amsterdam, the Netherlands

1

Speaker 


\section{Introduction}

The proposed high luminosity upgrade of the LHC (HL-LHC) run period will result in an integrated fluence larger than $1.5 \times 10^{15} \mathrm{n}_{\mathrm{eq}} / \mathrm{cm}^{2}$ for the innermost layer of silicon strip sensors in the CMS tracker. These fluence values are much higher than the radiation hardness limits of the present silicon strip sensors used in the CMS tracker [1]. To address this challenge, the CMS tracker collaboration initiated a systematic study of different silicon strip sensor design layouts consisting of different types of materials [2]. The effect of irradiation was investigated with a common measurement procedure and measurements were further complemented with simulations using Technology Computer Aided Design (TCAD) tools.

During sensor operation, radiation introduces defects, both in the silicon substrate causing bulk damage and at the $\mathrm{SiO}_{2} / \mathrm{Si}$ interface causing surface damage. Bulk damage produces adverse effects on detector operation by introducing deep trap levels of acceptor and donor type [3]. This leads to higher leakage current, lowering of charge collection efficiencies, and a change in the effective doping concentration of the substrate. On the other hand, surface damage can create a positive charge layer near the $\mathrm{SiO}_{2} / \mathrm{Si}$ interface and may create interface traps along the $\mathrm{SiO}_{2} / \mathrm{Si}$ interface [4]. A high value of oxide charge density $\left(\mathrm{Q}_{\mathrm{F}}\right)$ can create a sustainable accumulation electron layer under the $\mathrm{SiO}_{2} / \mathrm{Si}$ interface which can short the $\mathrm{n}^{+}$-strips in $\mathrm{n}^{+}$-p type sensors and can cause very high electric field near $\mathrm{p}^{+}$strips in $\mathrm{p}^{+}-\mathrm{n}$ type sensors. The accumulation layer provides a conduction channel and can spoil the position resolution in $\mathrm{n}^{+}-\mathrm{p}$ type sensors by lowering the inter-strip resistance $\left(\mathrm{R}_{\mathrm{int}}\right)$. In order to maintain the strip isolation two solutions are used: a p-spray layer or a p-stop pattern between strips, with very high doping densities [5-6]. In recent observations, p-stop/p-spray implants with very low doping densities of about $5 \times 10^{15} \mathrm{~cm}^{-3}$ appeared to maintain strip isolation between $\mathrm{n}^{+}$strips even after proton irradiation of above $1 \times 10^{15} \mathrm{n}_{\mathrm{eq}} / \mathrm{cm}^{2}$ [7]. Even more striking was the observation of good $\mathrm{R}_{\mathrm{int}}$ after proton irradiation with a fluence of about $7 \times 10^{14} \mathrm{n}_{\mathrm{eq}} / \mathrm{cm}^{2}$ for $\mathrm{n}^{+}-\mathrm{p}$ strip sensors, having no isolation structure at all [8].

The 2-D simulations of silicon strip sensors are carried out using Silvaco and Synopsys TCAD tools [9]. In TCAD simulations, an effective trap model (to approximate the known traps effects) for bulk damage is developed along with the suitable $Q_{F}$ values for surface damage. Higher $\mathrm{Q}_{\mathrm{F}}$ values are used for higher fluences with $\mathrm{Q}_{\mathrm{F}}$ saturation $\sim 2 \times 10^{12} \mathrm{~cm}^{-2}$.

\section{Combined bulk and surface damage model for TCAD simulations}

The Silvaco and Synopsys TCAD tools use slightly different physics models for radiation damage simulations and thus, can lead to different results for a given damage model [9]. Hence, separate bulk damage models are developed for Silvaco and Synopsys TCAD tools.

\subsection{Bulk damage model for Silvaco TCAD}

Initially, an effective two trap model for bulk damage was considered for the Silvaco TCAD tool [10]. But $\mathrm{C}_{\text {int }}$ (interstrip capacitance) and $\mathrm{R}_{\mathrm{int}}$ simulations using a two trap model reproduce the experimental measured $C_{i n t}$ and $R_{\text {int }}$ values only for very low $Q_{F}$ values [10-11]. 
Hence, an extended trap model with two more acceptor levels with higher introduction rates and one more donor was considered. The various parameters for this trap model are listed in table 1. This trap model produces leakage currents similar to measurements, as shown in figure 1 (left). The higher acceptor density creates dense negative space charge near $\mathrm{n}^{+}$strips. Due to this dense negative space charge, formation of an electron accumulation layer is suppressed which, otherwise, is formed due to surface damage. This effect of bulk damage on $\mathrm{R}_{\text {int }}$ between $\mathrm{n}^{+}$strips for $\mathrm{n}^{+}$-p strip sensor can be visualized in figure 1 (right). Here, $\mathrm{R}_{\text {int }}$ simulations are carried out for different fluences using $Q_{F}=5 \times 10^{11} \mathrm{~cm}^{-2}$. The simulate $R_{\text {int }}$ values are very low for fluence $=$ 0 , but improve with increase in fluence. The significant improvement of $R_{\text {int }}$ for higher fluences is due to the effect of bulk damage on the electron accumulation layer. This extended trap model reproduces the measured $\mathrm{R}_{\text {int }}$ and $\mathrm{C}_{\mathrm{int}}$ behaviour along with full depletion voltages, for both of the $\mathrm{n}^{+}-\mathrm{p}$ and $\mathrm{p}^{+}-\mathrm{n}$ strip sensors [11].

\begin{tabular}{|l|l|l|l|l|}
\hline Trap & Energy Level $(\mathrm{eV})$ & $\begin{array}{c}\text { Concentration } \\
\left(\mathrm{cm}^{-3}\right)\end{array}$ & $\sigma_{\mathrm{e}}\left(\mathrm{cm}^{2}\right)$ & $\sigma_{\mathrm{h}}\left(\mathrm{cm}^{2}\right)$ \\
\hline Acceptor & $\mathrm{E}_{C}-0.525$ & $3.0 \mathrm{x} \phi$ & $1 \times 10^{-14}$ & $1.4 \times 10^{-14}$ \\
\hline Acceptor & $\mathrm{E}_{C}-0.45$ & $40 \mathrm{x} \phi$ & $8 \times 10^{-15}$ & $2 \times 10^{-14}$ \\
\hline Acceptor & $\mathrm{E}_{C}-0.40$ & $40 \mathrm{x} \phi$ & $8 \times 10^{-15}$ & $2 \times 10^{-14}$ \\
\hline Donor & $\mathrm{E}_{V}+0.50$ & $0.6 \mathrm{x} \phi$ & $4 \times 10^{-15}$ & $4 \times 10^{-15}$ \\
\hline Donor & $\mathrm{E}_{V}+0.45$ & $20 \mathrm{x} \phi$ & $4 \times 10^{-15}$ & $4 \times 10^{-15}$ \\
\hline
\end{tabular}

Table 1. Bulk damage model used in the Silvaco simulations. $E_{C}$ and $E_{V}$ are energy of conduction and valence band respectively, while $\sigma_{\mathrm{e}}$ and $\sigma_{\mathrm{h}}$ are electron and hole capture cross sections. Here, $\phi$ is symbol for fluence in unit of $n_{\mathrm{eq}} / \mathrm{cm}^{2}$.
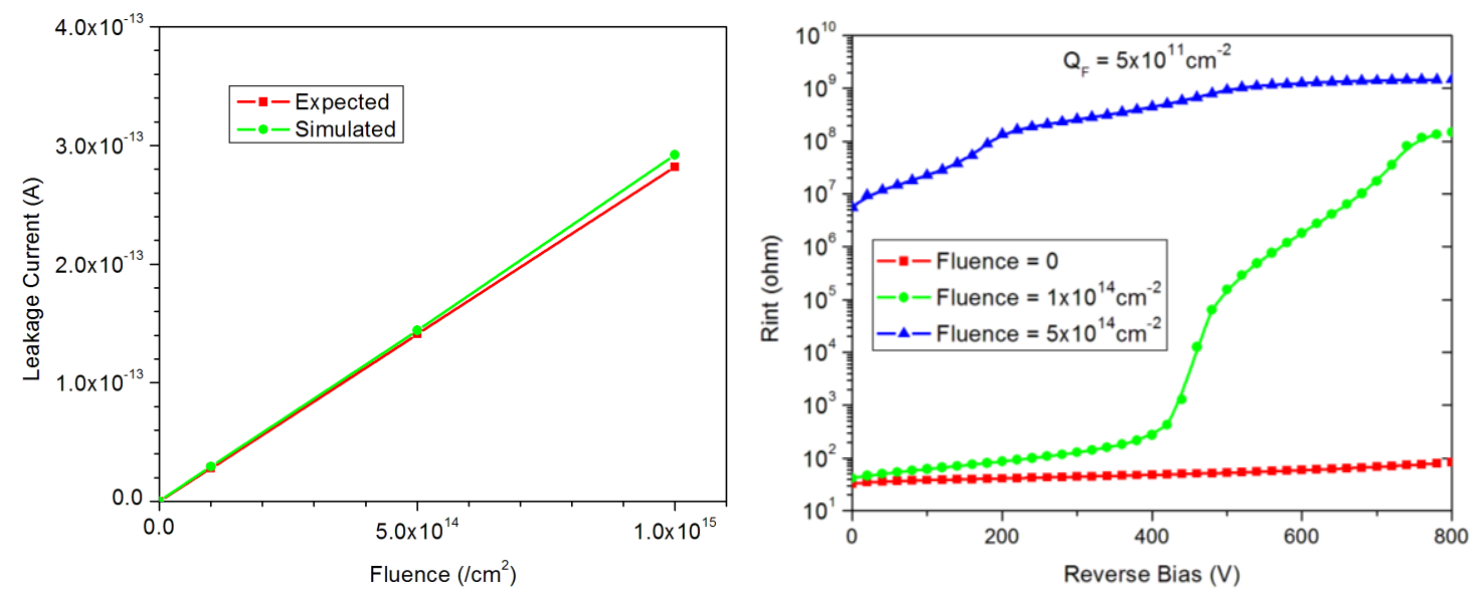

Figure 1. Comparison of simulated and expected (calculated from the damage coefficient) leakage current for different fluences for a diode of dimension $1 \mathrm{x} 1 \mathrm{x} 300 \mu \mathrm{m}^{3}$ using the extended trap model (left). $R_{\text {int }}$ vs. reverse bias voltage for $\mathrm{n}^{+}$-p strip sensors for different fluences for $\mathrm{Q}_{F}$ $=5 \times 10^{11} \mathrm{~cm}^{-2}$ (right). $R_{\text {int }}$ values are normalized for strip length equal to $1 \mathrm{~cm}$. Double $\mathrm{p}-$ stop structure with peak doping of $5 \times 10^{15} \mathrm{~cm}^{-3}$ is used between $\mathrm{n}^{+}$strips. 


\subsection{Bulk damage model for Synopsys TCAD}

For Synopsys TCAD simulations, separate trap models for proton and neutron damage were devised [9]. But similar to the Silvaco two trap model, the proton model was not able to account for the measured $\mathrm{R}_{\text {int }}$ and $\mathrm{C}_{\text {int }}$ trends for irradiated strip sensors. A new acceptor level with higher introduction rate was added to the proton model. In order to reproduce correctly Transient Current Technique (TCT) measurements in the simulation, the new acceptor level was used only within a few $\mu \mathrm{m}$ of the $\mathrm{SiO}_{2} / \mathrm{Si}$ interface [12] otherwise the signal is damped by the high trap concentration. The high acceptor density near the $\mathrm{SiO}_{2} / \mathrm{Si}$ interface is able to suppress electron accumulation layer between $\mathrm{n}^{+}$strips. This leads to higher $\mathrm{R}_{\text {int }}$ for irradiated $\mathrm{n}^{+}-\mathrm{p}$ strip sensor. The trap parameters for the three level trap model are listed in table 2.

\begin{tabular}{|c|l|c|l|l|}
\hline Trap & $\begin{array}{c}\text { Energy } \\
\text { Level }(\mathrm{eV})\end{array}$ & Concentration $\left(\mathrm{cm}^{-3}\right)$ & $\sigma_{\mathrm{e}}\left(\mathrm{cm}^{2}\right)$ & $\sigma_{\mathrm{h}}\left(\mathrm{cm}^{2}\right)$ \\
\hline Acceptor & $\mathrm{E}_{C}-0.525$ & $1.189 \times \phi+6.454 \times 10^{13}$ & $1 \times 10^{-14}$ & $1 \times 10^{-14}$ \\
\hline $\begin{array}{c}\text { Acceptor (used only within } \\
2 \mu \mathrm{m} \text { of the } \mathrm{SiO}_{2} / \mathrm{Si} \text { interface) }\end{array}$ & $\mathrm{E}_{C}-0.40$ & $40 \times \phi$ & $8 \times 10^{-15}$ & $2 \times 10^{-14}$ \\
\hline Donor & $\mathrm{E}_{V}+0.48$ & $5.598 \times \phi-3.959 \times 10^{14}$ & $1 \times 10^{-14}$ & $1 \times 10^{-14}$ \\
\hline
\end{tabular}

Table 2. Bulk damage model used in the Synopsys simulations.

The charge collection efficiency (CCE) simulations using Synopsys bulk damage models is in very good agreement with the measured values [9] as shown in figure 2. The CCE simulations reproduced the measured CCE loss between the strips for irradiated strips sensors also [12].

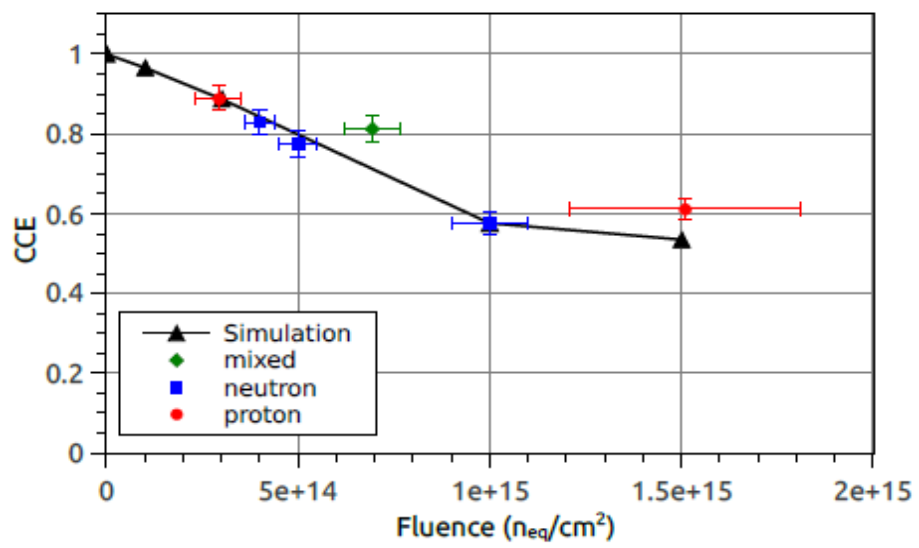

Figure 2. Comparison of simulated and measured CCE for different fluences using proton model and $\mathrm{Q}_{\mathrm{F}}=1 \times 10^{12} \mathrm{~cm}^{-2}$ at $253 \mathrm{~K}$ [9].

\section{Summary and conclusion}

In earlier simulation studies, surface damage and bulk damage were treated separately. But for strip sensors that are irradiated with hadrons both effects are taking place and should be included together in simulations. Further, the simulation models should be able to account for 
the surface as well as bulk properties of silicon strip sensors. With the extended models in Silvaco and Synopsys TCAD tools, $\mathrm{R}_{\mathrm{int}}, \mathrm{C}_{\mathrm{int}}$ as well as CCE can be reproduced for sensors up to fluences of $1.5 \times 10^{15} \mathrm{n}_{\mathrm{eq}} / \mathrm{cm}^{2}$. These models can be used for the prediction of radiation damage for sensors for the HL-LHC. Further work to improve the bulk damage models is ongoing.

\section{References}

[1] CMS collaboration, Technical proposal for the upgrade of the CMS detector through 2020, CERNLHCC-2011-006, CERN, Geneva Switzerland (2010).

[2] K.-H. Hoffmann et al., Campaign to identify the future CMS tracker baseline, Nucl. Instrum. Meth. A 658 (2011) 30.

[3] RD50 collaboration, Status reports, http://cern.ch/rd50/.

[4] J. Zhang et al., Investigation of X-ray induced radiation damage at the Si-SiO2 interface of silicon sensors for the European XFEL, arXiv:1210.0427 [physics.ins-det].

[5] C. Piemonte, Device simulations of isolation techniques for silicon microstrip detectors made on ptype substrates, IEEE Trans. Nucl. Sci. NS-53 (3) (2006) 1694.

[6] G. Verzellesi et al., Compact modeling of n-side interstrip resistance in $p$-stop and p-spray isolated double sided silicon microstrip detectors, IEEE Nuclear Science Symposium Conference (2000).

[7] A. Dierlamm, Characterisation of silicon sensor materials and designs for the CMS Tracker Upgrade, POS (Vertex 2012) 016.

[8] Y. Unno et al., p-Bulk silicon microstrip sensors and irradiation, Nucl. Instrum. Methods A 579 (2007) 614.

[9] R. Eber, Investigations of new Sensor Designs and Development of an effective Radiation Damage Model for the Simulation of highly irradiated Silicon Particle Detectors, PhD thesis (2013), KIT, Germany, IEKP-KA/2013-27

[10] T. Eichhorn, Silicon strip sensor simulations for the CMS phase-II tracker upgrade, IEEE, Nucl. Sci. Symp. (NSS/MIC) N2S-S (2012).

[11] R. Dalal et al., Combined effect of bulk and surface damage on strip insulation properties of proton irradiated $n^{+}-p$ silicon strip sensors, JINST 9 P04007, 2014.

[12] T. Peltola et al., A method to model the accumulation of oxide charge with fluence in an irradiated MSSD, $24^{\text {th }}$ RD50 Workshop, June, 11-13, 2014 Bucharest, Romania. 